\title{
HIPOTIROIDISMO SUBCLÍNICO EN NIÑOS Y ADOLESCENTES: SU IMPORTANCIA
}

\section{Subclinical hypothyroidism in children and adolescents: its importance}

\section{Dr. Francisco Carvajal Martínez*, Dra. Silvia Marín Juliá*, Dr. José María Basuan*, Dra. Yuraimi Piz Ramos*, Dr. José Rafael Gómez Hernández** y Dra. Julieta García Sáez***}

Recibido: 24/4/2018 Aprobado: 30/5/2018

Cómo citar: Carvajal-Martínez F, Marín-Juliá S.Basuan J, Gómez-Hernández J, García-Sáez J, Hipotiroidismo subclínico en niños y adolescentes: su importancia Ciencia y Salud [Internet]. 2018 mayo 1; [citado 2018 agosto 15]; 2(2):[aprox. 0 p.]. Disponible en: https://revistas.intec.edu.do/index.php/cisa/article/view/1181

\section{Resumen}

El Hipotiroidismo Subclínico (HSC) es una afección frecuente en edades pediátricas. El objetivo de este trabajo es presentar una revisión de los estudios realizados en los últimos años. Se destaca que el HSC es una entidad benigna, que frecuentemente remite y que habitualmente no necesita el tratamiento con Levotiroxina sódica (LT4). Finalmente, se señalan las posibles indicaciones de tratamiento con LT4.

Palabras clave: hipotiroidismo subclínico; niños y adolescentes; historia natural; tratamiento.

\footnotetext{
*Departamento de Endocrinología Pediátrica. Instituto Nacional de Endocrinología, Cuba

**Servicio de Endocrinología Pediátrica, Hospital Pediátrico, Pinar del Río

***Servicio de Endocrinología Pediátrica, Hospital Pediátrico Villa Clara. Email: endoped@infomed.sld.cu
}

\begin{abstract}
Subclinical Hypothyroidism ( $\mathrm{SH}$ ) is frequent in pediatric ages. The objective of this study is to present a review of the studies in the last years. It is emphasized that the $\mathrm{SH}$ is a benign entity; frequently it is not necessary a treatment with Synthetic levothyroxine (L-T4). The possible indications of LT4 treatment are established.
\end{abstract}

Keywords: Subclinical Hypothyroidism; children and teenagers; natural history; treatment. 


\section{Introducción}

El hipotiroidismo subclínico (HSC) es definido bioquímicamente como una elevación de las concentraciones séricas de la Hormona Estimulante del Tiroides (TSH) por encima de los niveles estadísticos normales, definidos en los valores de referencia, y las concentraciones de tiroxina libre (fT4) dentro del rango normal de referencia, esto según los autores Surks et al. ${ }^{1}$ y Cooper $^{2}$.

A lo largo de los años, se han utilizado otros sinónimos para definir esta entidad: hipotiroidismo ligero, hipotiroidismo preclínico, hipotiroidismo bioquímico, reserva tiroidea disminuida, fallo tiroideo ligero y disfunción tiroidea subclínica. Algunos autores, como Pearce ${ }^{3}$, han insistido en que es frecuente encontrar los valores de TSH > $4.2 \mathrm{mUI} / \mathrm{l}$; igualmente, se ha aconsejado basarse en los resultados de una segunda determinación (TSH y T4) para el diagnóstico y se diferencian dos categorías con relación a los niveles de TSH: niveles moderadamente elevados de TSH (4,0-10 UI/ml) y niveles más intensamente elevados (más de $10 \mathrm{UI} / \mathrm{ml}$ ).

Según Canaris ${ }^{4}$, Parle y Hollowell ${ }^{6}$ et al., la prevalencia del HSC en poblaciones adultas es mucho mayor que en edades pediátricas, aceptándose alrededor del 10\%, mientras en la infancia ese valor es menor. Se ha señalado que es más frecuente en el sexo femenino, así como en los pacientes de piel blanca.

\section{Desarrollo}

Se ha señalado que la prevalencia en la población pediátrica es menor al $2 \%$; así lo corrobora Bona ${ }^{7}$, aunque los estudios en estas edades son escasos. En la población adulta el HSC está asociado frecuentemente con riesgo de progresión de la afección hacia un hipotiroidismo total; se presenta en ocasiones con trastornos lipídicos, incremento del riesgo de aterosclerosis y está relacionado con aumento de la mortalidad debido a enfermedades cardiovasculares $^{8}$; sin embargo, las manifestaciones clínicas señaladas en la literatura en edades pediátricas son inconsistentes y la afección cursa frecuentemente asintomática en la niñez y la adolescencia.

Igualmente, se destaca la presencia de HSC en diferentes situaciones como: tiroiditis de Hashimoto, diabetes mellitus tipo 1, cirrosis biliar primaria, vitíligo, esclerosis sistémica progresiva, Síndrome de Down, Síndrome de Turner, enfermedad celíaca, anemia hemolítica autoinmune, enfermedad de Addison, hipotiroidismo congénito (durante el tratamiento insuficiente), medicamentos: yodo, amiodarona, litium, carbamazepina, etc.

No debemos olvidar que el valor de la elevación de la TSH es un indicador precoz y sensible de la función tiroidea. Se eleva antes de que aparezcan los síntomas y los signos de un hipotiroidismo y de que desciendan los niveles de t4 libre. Los factores de riesgo de fallo tiroideo son: historia familiar y personal de enfermedades tiroideas, presencia de anticuerpos antitiroideos positivos, tratamiento con radiaciones a zonas del cuello, la cabeza o el tórax, otras enfermedades autoinmunes, medicamentos como el yodo, el litium, etc., estar en la ancianidad y ser del sexo femenino.

Con relación al HSC en niños y adolescentes, se insiste en algunos aspectos:

Crecimiento y desarrollo: se conoce la importancia de las hormonas tiroideas en el crecimiento y en el desarrollo, así como su afectación en el hipotiroidismo clásico en estas edades. Eso explica la necesidad de conocer cómo es el crecimiento y el desarrollo de los pacientes con HSC. Aunque existen muy pocos estudios en este sentido, Cerbone et al. ${ }^{9}$ analizaron el crecimiento y el desarrollo en pacientes con HSC no tratados, encontrando que la talla y la edad ósea según la edad cronológica fueron normales. En un pequeño grupo se encontró baja talla secundaria, según estos autores, por causa familiar y por retraso constitucional del desarrollo, aunque señalan que no se deterioraron 
los parámetros de crecimiento evaluados durante el seguimiento de estos pacientes.

Por otro lado, Di Manse et al. ${ }^{10}$, al evaluar el posible efecto sobre la maduración ósea en pacientes con HSC no tratados, encontraron que los niveles de TSH al inicio del estudio y durante el seguimiento de estos pacientes no tuvo un impacto significativo sobre la densidad mineral ósea. Consideramos que se pudiera resumir que en los pocos estudios realizados no se ha demostrado afectación del crecimiento y ni del desarrollo por el HSC sin tratamiento.

HSC y migrańa: existen pocos estudios que han evaluado este aspecto (solo dos), y son contradictorios. Fallah et al. ${ }^{11}$, evaluando la prevalencia mensual de cefaleas en pacientes con HSC, concluyó que los pacientes entre 5 y 15 años de edad sufren migraña de manera más frecuente que en etapas posteriores de la vida y opinan que sería aconsejable la evaluación de la función tiroidea en ellos. Sin embargo, estos hallazgos no fueron confirmados por Ekici y Cebeci (citado por Gawlik A et al. ${ }^{7}$ ), que solo detectaron HSC en el $5 \%$ de los pacientes con migraña, por lo que concluyen que la evaluación inicial por screening para HSC no es necesaria. A pesar de esto, consideramos que todavía se debe esperar que se realicen más estudios en esta dirección para poder llegar a una decisión adecuada.

HSC y desarrollo intelectual: existen muy pocos estudios que evalúen esta esfera en pacientes en edades pediátricas con HSC. Cerbone et al. ${ }^{9}$ no encontraron relación alguna entre los niveles de TSH y los coeficientes de inteligencia (ejecutiva, verbal y total); asimismo, Ergur et al. ${ }^{12}$ no encontraron alteraciones en la función cognitiva. Al parecer, por los pocos estudios realizados no existe afectación a nivel del desarrollo intelectual en los pacientes con HSC.

HSC y alteraciones cardiovasculares: en este aspecto se ha profundizado un poco más. Ittermann et al. ${ }^{13}$ evaluaron la relación entre las concentraciones séricas de TSH y la tensión arterial sistólica y diastólica en 12,353 pacientes; el HSC se encontró solo en el 5\% de ese estudio y concluyeron que existe en este grupo de pacientes el riesgo de hipertensión. Aunque no encontraron una explicación, opinan que quizás puede ser debido a una disfunción endotelial, hipertrofia ventricular izquierda o afectación de las paredes arteriales.

Por otro lado, Cerbone et al. ${ }^{9}$ evaluaron los factores de riesgo cardiovascular y clínico en un grupo de pacientes con HSC, concluyendo que el HSC ligero de largo plazo puede estar asociado con anormalidades pro-aterogénicas, aunque estos cambios pueden no representar etapas tempranas de ateroesclerosis.

Por su parte, Chen et al..$^{14}$, al estudiar en una población china si existía relación entre los niveles de TSH y la presión arterial, observaron que en los pacientes con HSC, evaluados durante 7 meses, los niveles de presión sistólica y diastólica (los Z score) eran más altos que en el grupo control.

Al parecer, por los estudios señalados, en la población pediátrica y adolescente con HSC deben ser seguidos estos aspectos con mucho cuidado, aunque consideramos que los estudios que así lo indican son escasos.

HSC y vitiligo: solo conocemos un trabajo, citado por Gawlik et al. ${ }^{7}$, en el que se investigó la relación entre estas dos afecciones, sin encontrar ninguna relación entre alteraciones de la función tiroidea en comparación con los controles.

HSC y pubertad: Cerbone et al. ${ }^{9}$ no detectaron alteraciones de la pubertad en el grupo de pacientes con HSC no tratados; por su lado, otros investigadores, como Rapa et al. ${ }^{15}$, no observaron relación entre las concentraciones de TSH y el estado puberal, determinado por inspección visual, según los estadios de Tanner. Por lo antes señalado, al parecer no existe relación entre el HSC y alguna alteración de la pubertad. 
HSC y nińos nacidos con fertilización in vitro: se han realizado dos investigaciones en estos niños evaluando la función tiroidea. Onal et al. ${ }^{16}$ analizaron la función tiroidea a la edad de 2-4 semanas, examinaron 98 niños concebidos por fertilización in vitro; de estos, 10 tuvieron la TSH con valores mayores de $6.5 \mathrm{mUI} / \mathrm{L}$ y fueron diagnosticados como HSC; se compararon con 10 nińos nacidos de forma normal, pero con hipertirotropinemia, quienes estaban eutiroideos. A todos se les realizó el test de TRH y se pudo constatar que los nińos nacidos a través de fertilización in vitro tenían los valores de TSH significativamente más elevados que el grupo control.

Por su parte, Sakka et al. ${ }^{17}$ estudiaron a 106 niños nacidos por fertilización in vitro, con edades entre 4 y 14 años, además de un grupo control, y encontraron niveles elevados de TSH en 7 niños, para orientar en estos el diagnóstico de HSC; sin embargo, no se constató este diagnóstico en ninguno de los controles. Estos autores concluyeron que el HSC observado en los pacientes con antecedentes de nacimiento a través de la fertilización in vitro no tenía ninguna relación con diversos factores como son: edad gestacional, edad materna, tamaño pequeño para la edad gestacional, etc.

\section{Historia natural del HSC}

Lazar et al..$^{18}$ estudiaron la historia natural de las pruebas de la función tiroidea durante 5 años en un gran estudio, analizando a 121,052 niños con HSC entre 0.5-16 años. Ellos concluyeron que: las cifras de TSH ligeramente elevadas o normales al inicio frecuentemente se mantienen normales o se normalizan espontáneamente sin necesidad de tratamiento; además, que los pacientes con niveles elevados de TSH mayor de $7.5 \mathrm{mUI} / \mathrm{L}$, particularmente si son hembras, tienen un mayor riesgo de persistir con concentraciones elevadas de TSH.

Wasniewska et al. ${ }^{19}$, por su parte, evaluaron el curso natural en pacientes con HSC, a los 6, 12 y 24 meses.
Encontrando que todos los pacientes eran asintomáticos, sin cambios en la talla o en la masa corporal durante su seguimiento; asimismo, el 41,3\% de los pacientes normalizaron los valores de la TSH en su seguimiento, solo el $12 \%$ observó un incremento de las cifras de TSH (valores entre 10-15 mUI/L).

En ocasiones, el HSC se observa en el transcurso del cuadro clínico de una tiroiditis autoinmune. Zois et al. ${ }^{20}$ señalaron la persistencia del HSC en esta afección en 7 niños griegos seguidos por 5 años, encontrando que la hipoecogenicidad y los anticuerpos antitiroideos se incrementaron en ese tiempo. Por su parte, Radetti et al. ${ }^{21}$, al estudiar 55 niños con tiroiditis de Hashimoto, encontraron que 39 de ellos tuvieron HSC, y de estos el $29.1 \%$ tuvo TSH constantemente elevada.

Bona et al. ${ }^{7}$, evaluando la mayoría de los estudios publicados, enfatiza que el HSC en niños y adolescentes es un proceso benigno que puede regresar; asimismo, que existe bajo riesgo de que evolucione hacia un hipotiroidismo total. Ampliando lo anterior, Radetti et al. ${ }^{21}$ han señalado que la presencia inicial de bocio y anticuerpos antitiroideos elevados, así como la progresión de estos anticuerpos y de los valores de TSH, pueden predecir la evolución hacia un hipotiroidismo total.

También, se ha encontrado HSC en "falsos positivos" durante el screening de hipotiroidismo congénito. Leonardi et al. ${ }^{22}$, al evaluar su serie en niños italianos, observaron que en la mitad de las pacientes la TSH se normalizó y en la otra mitad se mantuvo el HSC; a los tres años de evolución, ninguno de los niños había tenido hipotiroidismo total. Además, encontraron en algunos pacientes alteraciones morfológicas tiroideas, como la hipoplasia. Estos autores consideraron que este cuadro de HSC pudiera deberse a anomalías anatómicas o funcionales de la glándula tiroidea, aunque su significado clínico no está claro, ya que ninguno de esos niños desarrolló un hipotiroidismo total durante su seguimiento. 
Igualmente, se ha señalado que el HSC debe ser diferenciado al aumento transitorio o fisiológico de la TSH, especialmente durante la fase de recuperación de una enfermedad no tiroidea o en la etapa posterior de una tiroiditis subaguda, por lo que se plantea que en estas situaciones se debe reevaluar los valores de TSH después de 3-6 meses.

\section{Evolución de los valores de TSH durante el HSC en nińos y adolescentes}

Lo habitual es que disminuyan las concentraciones de TSH; sin embargo, en muy pocos casos existe incremento y está asociado a: presencia de bocio o de anticuerpos antitiroideos positivos o incrementos de estos o del bocio ${ }^{21,23}$. También, se ha señalado que puede existir reversibilidad espontánea de las concentraciones de TSH entre el 41-73\% de los sujetos evaluados ${ }^{18,19}$.

Clásicamente, se ha orientado el tratamiento con LT4, aunque la decisión de tratar o no debería estar basada en el riesgo de evolución hacia un hipotiroidismo total y a las consecuencias de la hipertirotropinemia "per se". En los adultos se ha observado diferentes efectos: depresión, incremento de las afecciones cardiovasculares, dislipidemia, etc. No obstante, en niños y adolescentes no es así, pues habitualmente esta afección es asintomática y frecuentemente reversible. A pesar de esto, se debe tener cuidado por los efectos adversos del sobretratamiento con LT4, lo cual puede originar desórdenes cardiovasculares y disminución de la densidad mineral ósea, hipertiroidismo, entre otros.

Bona et al. ${ }^{7}$, evaluando los pocos estudios realizados en esta dirección, llegaron a esa conclusión ya que el HSC en edades pediátricas es una condición limitada, con baja frecuencia de progresión al hipotiroidismo total; consideran que solo se debe valorar iniciar el tratamiento con LT4 en las siguientes situaciones: valores de TSH mayores a 10 $\mathrm{mUI} / \mathrm{L}$, cuando los síntomas y signos de fallo de la disfunción tiroidea o la presencia de bocio son detectados o cuando el HSC está asociado a otras enfermedades crónicas. En niños con HSC sin bocio con anticuerpos antitiroideos negativos y valores de TSH entre 5-10 mUI/L el tratamiento con LT4 no está justificado. Monzani et al. ${ }^{24}$ consideran que si existe baja talla o fallo de la velocidad de crecimiento también debe valorarse el tratamiento con LT4.

Asimismo, Gawlik et al. ${ }^{8}$, al evaluar la mayoría de los pocos trabajos publicados sobre HSC en niños y adolescentes, consideran que los pocos síntomas reportados no tienen conexión de forma obligada con las concentraciones elevadas de TSH. Mientras que, tras haber realizado un estudio con pacientes adultos, Abu-Helalah et al. ${ }^{23}$ consideran que el tratamiento con LT4 en esas edades mejora solo en un $1 \%$ la calidad de vida.

Como conclusión sobre si se debe o no tratar, se debería valorar "no tratar" a pacientes sin bocio, sin anticuerpos antitiroideos positivos, con valores de TSH entre 5 y $10 \mathrm{mUI} / \mathrm{L}$; igualmente debemos recordar que la reversibilidad espontánea de la TSH elevada puede ocurrir entre el $4 \%$ y el $73 \%$ de los pacientes.

En contraste con lo antes dicho, debemos valorar "tratar" en las siguientes situaciones: asociación con baja talla o con velocidad de crecimiento lenta, presencia o incremento del bocio, niveles de TSH > $7.5 \mathrm{mUI} / \mathrm{L}$, sexo femenino, presencia de anticuerpos antitiroideos positivos, asociación a otras enfermedades crónicas como la enfermedad celíaca y la presencia del HSC en la época de lactante-neonatal.

\section{Hipotiroidismo subclínico con obesidad}

Hemos señalado que el HSC en edades pediátricas no es frecuente, o sea, menos del 10\% (más cercano al 2\%); sin embargo, cuando evaluamos esta afección en niños con HSC, pero con sobrepeso u obesos se señala que incrementa su diagnóstico con una frecuencia de HSC alrededor del 30\% $\%^{25-33}$. 
Las hormonas tiroideas están relacionadas, de forma importante, con la homeostasis energética, la regulación del metabolismo y con el peso corporal. Así, uno pudiera llegar a la opinión clásica de que en el hipotiroidismo existe aumento del peso; y que, en su sentido contrario, o sea, en el hipertiroidismo, se observa la disminución del peso corporal. Pero, el mecanismo interno de las alteraciones de la función tiroidea observada en la obesidad todavía es incierta, por lo que esta alteración pudiera ser consecuencia más que causa del exceso de peso.

En el obeso, el HSC es más frecuente, constatándose valores elevados de TSH y valores normales de T4 total (tT4) y T4 libre (fT4); a pesar de esto, se han encontrado cambios en los valores de T3 libre (fT3) y de T3 reversa (rT3), confirmándose cifras elevadas en el paciente obeso con HSC. Por su parte, Marras et al. ${ }^{30}$, evaluando la función tiroidea en niños y adolescentes obesos, encontraron que menos del $1 \%$ de los valores del índice de masa corporal es explicado por la TSH, por lo que concluyen que las anormalidades de las hormonas tiroideas no están tan relacionadas con la causa de la obesidad.

En otro sentido, diversas hipótesis se han señalado con la finalidad de tratar de explicar los mecanismos de la hipertirotropinemia en el paciente obeso. La teoría adaptativa establece que las hormonas tiroideas aumentan para incrementar el gasto energético y así disminuir la obesidad; se plantea que este proceso pudiera estar mediado por la leptina, ya que se ha comprobado que los obesos tienen niveles más elevados de esta hormona, la cual podría estimular directamente la secreción de TSH.

También se ha dicho que la leptina pudiera estimular la conversión de T4 a T3, lo que se interpretaría como un mecanismo para favorecer la pérdida de peso, ya que la disminución de T4 estimularía la producción de TSH. Al parecer, las concentraciones de leptina son proporcionales a la masa grasa corporal y aumentan en la obesidad, así como hay sincronicidad de la secreción de leptina y TSH en nińos y en adultos. Igualmente, es probable que la leptina pueda modificar la secreción hipotalámica de TRH directa o indirectamente; de esta forma estimularía la secreción de TRH, lo que favorecería, a su vez, la secreción de TSH.

La teoría inflamatoria plantea que las citoquinas pro-inflamatorias producidas por el tejido adiposo podrían disminuir la producción periférica de hormonas tiroideas. Las citoquinas bloquean la captación de yodo por el tiroides, provocando un aumento compensatorio de TSH. En adición, se ha observado que los niveles de T3 disminuyen, así como los niveles de TSH, después de la pérdida de peso $^{33}$; los cambios del estilo de vida y el incremento del ejercicio físico favorecen esta situación. Quizás la pérdida de peso o la modificación de la composición corporal reducen el estado de inflamación que se observa en el paciente obeso, expresado por la disminución de las citoquinas.

En síntesis, si es necesario el tratamiento con levotiroxina en el HSC en el niño o el adolescente obeso, partiendo de la base de que estas alteraciones son funcionales ${ }^{33}$ (Longhi S., 2013) y que frecuentemente revierten a la normalidad después de que se logre la reducción de peso, no sería necesario indicar el tratamiento.

\section{Conclusiones}

El HSC en edades pediátricas no es frecuente, es una afección benigna y comúnmente los valores de TSH revierten a la normalidad sin tratamiento. Empero, es importante realizar los estudios de anticuerpos antitiroideos, además de evaluar la función hormonal. Conviene señalar que el HSC puede ser observado en numerosas afecciones.

\section{Bibliografía}

1. Surks MI et al. Subclinical thyroid disease: scientific review and guidelines for diagnosis 
and management. Journal of the American Medical Association. 2004; 291:228-238.

2. Cooper DS, Biondi B. Subclinical thyroid disease. Lancet. 2012; 379:1142-1154.

3. Pearce HS, Brabant G, Duntas L, Monzani F, Peeters R, Razvi $S$ et al. European Thyroid Association guideline of management of subclinical hypothyroidism. Eur Thyroid J. 2013; 2:215-28.

4. Canaris $G$ et al. The Colorado thyroid disease prevalence study. Archives of Internal Medicine 2000; 160:526-534.

5. Parle JV et al. Prevalence and follow-up of abnormal thyrotrophin (TSH) concentration in the erderly in the United Kingdom. Clinical Endocrinology. 1991; 34:77-83.

6. Hollowell JG etal. Serum TSH,T (4), and thyroid antibodies in the United States population (1988-1994). Journal of Clinical Endocrinology and Metabolism. 2002; 87:487-499.

7. Bona G, Prodam F, Monzani A. Subclinical hypothyroidism in children: Natural history and when to treat. J Clin Res Pediatr Endocrinol. 2013; 5(1):23-28.

8. Gawlik A et al. Subclinical hypothyroidism in children and adolescents: Is it clinically relevant? International Journal of Endocrinology. 2015; Article ID 691071:1-12.

9. Cerbone Metal. Linear growth and intellectual outcome in children with long-term idiopathic subclinical hypothyroidism. European Journal of Endocrinology. 2011; 164(4):591-597.

10. Di Manse R et al. Bone health in children with long-term idiopathic subclinical hypothyroidism. Italian Journal of Pediatrics. 2012; 1(38)

11. Fallah $\mathrm{R}$ et al. Frequency of subclinical hypothyroidism in 5-to 15-year-old children with migraine headache. Journal of Pediatric Endocrinology and Metabolism. 2012; 25(9-19):859-862.

12. Ergur AT et al. Neurocognitive functions in children and adolescents with hypothyroidism sub clinical. Journal of Clinical Research in Pediatric Endocrinology. 2012; 4(1):21-24.

13. Ittermann $T$ et al. Serum thyroid-stimulating hormone levels are associated with blood pressure in children and adolescents. Journal of Clinical Endocrinology Metabolism. 2012; 97(3):828-834.

14. Chen $\mathrm{H}$ et al. Investigation of thyroid function and blood pressure in school-aged subjects without overt thyroid disease. Endocrine. 2012; 41(1):122-129.

15. Rapa A et al. Sub clinical hypothyroidism in children and adolescents: a wide range of clinical, biochemical, and genetic factors involved. Journal of Clinical Endocrinology Metabolism. 2009; 94:2414-2420.

16. Onal $\mathrm{H}$ et al. Subclinical hypothyroidism in vitro fertilization babies. Acta Paediatrica. 2012; 101(6):248-252.

17. Sakka SD et al. Euthyroid hyperthyrotropinemia in children born after in vitro fertilization. Journal of Clinical Endocrinology and Metabolism. 2009; 94(4):1338-1341.

18. Lazar I et al. Natural history of thyroid function test overt 5 years in a large pediatric cohort. Journal of Clinical Endocrinology and Metabolism. 2009; 94(5):1678-1782.

19. Wasniewska M et al. Prospective evaluation of the natural course of idiopathic subclinical hypothyroidism in childhood and adolescents. European Journal of Endocrinology. 2009; 160(3): 417-421. 
20. Zois $C$ et al. Natural course of autoimmune thyroiditis after elimination of iodine deficiency in north western Greece. Thyroid. 2006; 16:289-293.

21. Radetti G et al. Study Group for Thyroid Disease of the Italian Society for Pediatric Endocrinology and Diabetes (SIEDP/ ISPED). The natural history of euthyroid Hashimoto's thyroiditis in children. J Pediatr. 2006; 149:827-832.

22. Leonardi et al. Longenital study of thyroid function in children with mild hyperthyrotropinemia at neonatal screening for congenital hypothyroidism. J. Clin Endocrinol Metab. 2008; 93:2679-2685.

23. Abu-Helalah $\mathrm{M}$ et al. A randomized double-blind crossover trial to investigate the efficacy of screening for adult hypothyroidism. J Med Sreening. 2010; 17:164-169.

24. Monzani A et al. Natural history of subclinical hypothyroidism in children and adolescents and potencial effects of replacement therapy: a review. European Journal of Endocrinology. 2013; 168: R1-R11.

25. Zaninovich A. Hormonas tiroideas, obesidad y termogénesis en grasa parda. Medicina (Buenos Aires). 2001; 61:597-602.
26. Reinehr T. Obesity and thyroid function. Molecular and celular endocrinology. 2010; 316:165-171.

27. Reinehr T, Andler W. Thyroid hormones before and after weight loss in obesity. Arch Dis Child 2002; 87:320-323.

28. Biondi B. Thyroid and obesity: An intriguing relationship. J Clin Endocrinol Metab. 2010; 95(8): 3614-3617.

29. Sánchez $T$ et al. Niveles de hormonas tiroideas en niños obesos. Rev Chil Pediatr. 2014; 85 (3):288-297.

30. Marras V et al. Thyroid function in obese children and adolescents. Horm Res Paediatr. 2010; 73:193-197.

31. Reinehr T. Thyroid function in the nutritionally obese child and adolescent, Current Opinion in Pediatrics 2011; 23:415-420.

32. Reinehr $\mathrm{T}$. et al. Thyroid hormones and their relation to weight status. Horm Res. 2008; 70:51-57.

33. Longhi $S$ y Radetti G. Thyroid function and obesity. J Clin Res Pediatr Endocrinol. 2013; 5(1): 40-44. 